\title{
Effect of the Healthy MOMs Lifestyle Intervention on Reducing Depressive Symptoms Among Pregnant Latinas
}

\author{
Edith C. Kieffer • Cleopatra H. Caldwell • \\ Diana B. Welmerink - Kathleen B. Welch • \\ Brandy R. Sinco $\cdot$ J. Ricardo Guzmán
}

Published online: 26 May 2012

(c) Society for Community Research and Action 2012

\begin{abstract}
Depression during the prenatal and postpartum periods is associated with poor maternal, perinatal and child outcomes. This study examines the effectiveness of a culturally and linguistically tailored, social support-based, healthy lifestyle intervention led by trained community health workers in reducing depressive symptoms among pregnant and early postpartum Latinas. A sample of 275 pregnant Latinas was randomized to the Healthy MOMs Healthy Lifestyle Intervention (MOMs) or the Healthy Pregnancy Education (control) group. More than one-third of participants were at risk for depression at baseline. MOMs participants were less likely than control group participants to be at risk for depression at follow-up. Between baseline and 6 weeks postpartum, MOMs participants experienced a significant decline in depressive symptoms; control participants experienced a marginally significant decline. For MOMs participants, most of this decline occurred during the pregnancy intervention period, a time when no change occurred for control participants. The change in depressive
\end{abstract}

E. C. Kieffer $(\bowtie) \cdot$ D. B. Welmerink · B. R. Sinco

School of Social Work, University of Michigan,

1080 S. University, Room 3770, Box 10, Ann Arbor,

MI 48109-1106, USA

e-mail: ekieffer@umich.edu

C. H. Caldwell

Department of Health Behavior and Health Education, School

of Public Health, University of Michigan, Ann Arbor, MI, USA

\section{K. B. Welch}

Center for Statistical Consulting and Research (CSCAR),

University of Michigan, Ann Arbor, MI, USA

J. R. Guzmán

Community Health and Social Services (CHASS) Center, Inc.,

Detroit, MI, USA symptoms during this period was greater among MOMs than control participants ("intervention effect"). From baseline to postpartum, there was a significant intervention effect among non-English-speaking women only. These findings provide evidence that a community-planned, culturally tailored healthy lifestyle intervention led by community health workers can reduce depressive symptoms among pregnant, Spanish-speaking Latinas.

Keywords Prenatal and postpartum depression .

Latinas · Community-based intervention .

Community health workers - Diabetes .

Community-based participatory research

\section{Introduction}

Depression during pregnancy is associated with fetal growth restriction, low birth weight and preterm birth (Diego et al. 2009; Gavin et al. 2009; Grote et al. 2010); and is a strong correlate of postpartum depression (Martinez-Schallmoser et al. 2003; Rich-Edwards et al. 2006; Robertson et al. 2004; Zayas et al. 2003). Both prenatal and postpartum depression have been linked to a variety of attachment and mood disorders and other poor emotional and physical health outcomes in mothers, newborns, infants and children (Marcus et al. 2011; Pearson et al. 2010; Perry et al. 2011; Wojcicki et al. 2011). The prevalence of moderate to high levels of depressive symptoms during and after pregnancy vary by population, screening instruments, criteria used, and timing of assessment. An estimated $20 \%$ of women are estimated to experience depression during the perinatal period (Gavin et al. 2005).

Relatively frequent contact with the health care system during pregnancy and the early postpartum period offers 
important opportunities to identify and reduce depressive symptoms (Lancaster et al. 2010). However, screening, referral and treatment for depressive symptoms are inadequate for many women (Flynn et al. 2010; Grote et al. 2009; Miranda et al. 2003), and many women do not seek or receive treatment (McGarry et al. 2009). For low income, racial and ethnic minority and immigrant women, barriers to access and use of mental health services may be greater. Among these are lack of mental health insurance coverage and providers; chronic life stressors; traumatic life events; priorities focused on attaining basic life needs; religious and cultural beliefs and practices, including stigma; conflicting time commitments; lack of child care and transportation; poor maternal, infant and family health; social and linguistic isolation; and fear of deportation (Grote et al. 2009; Levy and O'Hara 2010; McGarry et al. 2009; Miranda et al. 2003; Spinelli and Endicott 2003). Although some innovative interventions have been reported (Grote et al. 2009; Le et al. 2011; Levy and O'Hara 2010; Miranda et al. 2003; Muñoz et al. 2007; Spinelli and Endicott 2003), most current treatment programs do not adequately address these barriers to depression treatment, particularly for low income, immigrant Latinas. The current study examines the effectiveness of a culturally and linguistically tailored intervention, which provided social support for healthy lifestyles, in reducing depressive symptoms among pregnant and postpartum Latinas.

\section{Depression Among Latinas During the Pregnancy} and the Postpartum Periods

A recent large study in a multiethnic, urban population found that Hispanic ethnicity more than doubled the odds of depression during pregnancy, independent of other risk factors (OR 2.50; $95 \%$ CI 1.09-5.72) (Melville et al. 2010). Estimates of the prevalence of moderate to high levels of depressive symptoms among Latina women are generally between 30 and $40 \%$ during pregnancy (Davila et al. 2009; Fortner et al. 2011; Hromi-Fiedler et al. 2011; Jesse and Swanson 2007; Lara et al. 2009; MartinezSchallmoser et al. 2005; Martinez-Schallmoser et al. 2003). Among Puerto Rican and Dominican women in New York City, $53 \%$ were depressed in the third trimester (Zayas et al. 2003). Among Puerto Rican women in Harford, Connecticut, $31 \%$ were depressed, including $28.7 \%$ of women in the second trimester and $36.2 \%$ in the third trimester. Most studies find a progressive decline in depressive symptoms after childbirth, as the postpartum period progresses (Davila et al. 2009; Diaz et al. 2007; Perry et al. 2011; Yonkers et al. 2001; Zayas et al. 2003). Depression prevalence declined from 35 to $26 \%$ of postpartum Latinas in Dallas between 3 and 4 weeks postpartum (Yonkers et al. 2001). However, a study of
Mexican-American women in Chicago found an increase from $38 \%$ during pregnancy to $53 \%$ at 6 weeks postpartum (Martinez-Schallmoser et al. 2003). High levels of depressive symptoms were reported for $59 \%$ of postpartum Mexican-American women in a Northern California study (Heilemann et al. 2004).

\section{Risk Factors for Depression}

Prenatal depression is a very strong predictor of postpartum depression (Martinez-Schallmoser et al. 2003; RichEdwards et al. 2006; Robertson et al. 2004; Zayas et al. 2003). Other risk factors vary among studies. Pregnant and postpartum Latinas may be more likely to experience stressful life events, financial hardships and low socioeconomic status, each of which has been associated with postpartum depressive symptoms in varied populations (O'Hara 2009; Rich-Edwards et al. 2006). The effect of acculturation on depressive symptom prevalence was inconsistent among studies. Most frequently, greater levels of acculturation were associated with increased risk for depression among Latinas, as measured by US birthplace (Davila et al. 2009), low use of Spanish (MartinezSchallmoser et al. 2003), speaking both Spanish and English (Yonkers et al. 2001), and living in the US as a child (Heilemann et al. 2004).

Low levels of social support (Diaz et al. 2007; Kuo et al. 2004; Martinez-Schallmoser et al. 2003; Sleath et al. 2005; Surkan et al. 2006) and lack of satisfaction with social support (Martinez-Schallmoser et al. 2003) were associated with increased prenatal and postpartum depression risk. Being single was a risk factor for depression in two studies with Latinas (Davila et al. 2009; Hromi-Fiedler et al. 2011); living with a partner/spouse was protective in another (Fortner et al. 2011). Other risk factors for perinatal depression among Latinas included intimate partner violence and chronic medical conditions (Melville et al. 2010), household food insecurity, poor to fair health during pregnancy and primiparity (Hromi-Fiedler et al. 2011), low sense of mastery and life satisfaction (Heilemann et al. 2004).

Interventions to Reduce Depressive Symptoms During Pregnancy and the Postpartum Periods

Pregnancy and the postpartum period present important opportunities to prevent depressive symptoms and to identify and address depression that occurs (Flynn et al. 2010; Lancaster et al. 2010). Research has focused on strategies for identifying women at risk for depression and engaging them in psychotherapy, prescribing medication or both, and evaluating outcomes (Flynn et al. 2010; Givens et al. 2007; Grote et al. 2009; Le et al. 2011; Muñoz et al. 
2007; O'Hara 2009; Spinelli and Endicott 2003). Most non-biological interventions have used cognitive behavioral therapy (CBT) or interpersonal psychotherapy approaches to reduce depressive symptoms among women identified as high risk for depression during or after pregnancy (Dennis and Creedy 2004; O’Hara 2009).

Most depression intervention studies have reported results for non-Hispanic white women, African American women, or mixed ethnic populations, and some do not disaggregate findings by ethnic group (Grote et al. 2009; Miranda et al. 2003; O’Hara 2009; Roman et al. 2009). A recent study investigating postpartum depressive symptoms found that Latinas were at increased risk of not seeking help after reporting depressive symptoms compared to nonHispanic white women (McGarry et al. 2009). Four studies have reported results of bilingual, culturally tailored psychotherapy interventions, administered solely or primarily for pregnant or postpartum Latina women by trained health care providers (Beeber et al. 2010; Le et al. 2011; Muñoz et al. 2007; Spinelli and Endicott 2003).

Spinelli and Endicott (2003) studied 38 women with major depression during pregnancy, 25 of whom were immigrants from the Dominican Republic. Participants in the 16-week interpersonal psychotherapy intervention, conducted by trained and experienced psychotherapists, showed significant improvement in depressive symptoms compared to a control group that received a 16-week parenting education program (Spinelli and Endicott 2003). Results were not disaggregated by ethnic origin. In California, Muñoz et al. conducted a pilot study, Mamás y Bebés, with 41 pregnant, predominantly Mexican immigrant Latinas at high risk for major depressive episodes, using a CBT-based intervention consisting of a 12-week mood management course during pregnancy and four postpartum booster sessions (Muñoz et al. 2007). The 21 intervention participants had fewer major depressive episodes (14.5 vs. $25.7 \%$ ), but no difference in mean depressive symptoms scores, compared to the 20 control group participants. Small sample size may have limited the ability of this study to detect statistically significant results. Building upon this work, Le et al. modified Mamás y Bebés to include eight classes during pregnancy and three postpartum booster sessions for a predominantly immigrant Central American population of 217 women in Washington D.C. (Le et al. 2011). Control group participants received an 8-week pregnancy education course. Depressive symptoms decreased significantly from early pregnancy to 1 year postpartum in both groups. However, the decrease in depressive symptoms observed in intervention participants was not significantly different than the decrease observed in control group participants over time (Le et al. 2011). There was a significant intervention effect during pregnancy that did not persist postpartum.
Beeber et al. (2010) compared the outcomes of 71 Latina mothers with high levels of depressive symptoms, whose 6- to 18-month-old infants participated in an Early Head Start Program. Compared to usual care, the 16-session in-home interpersonal therapy program, conducted by trained psychiatric nurses, was associated with a significantly greater decrease in depressive symptoms immediately following the intervention and 1-month postintervention (Beeber et al. 2010).

In summary, a high percentage of Latinas experience depressive symptoms during pregnancy and the postpartum period, and often encounter many barriers to treatment. The few interventions conducted with Latinas provide models for using formal psychotherapeutic methods conducted by mental health professionals and students with women with, or at high risk for, major depression. These interventions did not attempt to reduce the risk of depression in a general population of pregnant and postpartum Latinas. While achieving progress in reducing barriers to participation, these studies had mixed success in reducing depressive symptoms, particularly in the early postpartum period, and had relatively small sample sizes.

\section{Background for the Current Study}

The current study is derived from Healthy Mothers on the Move (Healthy MOMs), a prospective randomized controlled clinical trial designed to reduce risk factors for obesity and type 2 diabetes. Healthy MOMs aimed to demonstrate the effectiveness of a healthy lifestyle intervention tailored to the needs of pregnant and postpartum Latino women. Healthy MOMs was planned, developed and implemented in Detroit, using a community-based participatory research (CBPR) approach in affiliation with the Detroit Community Academic Urban Research Center and the REACH Detroit Partnership (Israel et al. 2001; Kieffer et al. 2002, 2004, 2005; Thornton et al. 2006). It was guided by a steering committee of community resident women of childbearing age and representatives of community, academic and health-related organizations. Formative research included in-depth individual interviews and focus groups conducted with pregnant and postpartum Latinas and organization and policy leaders in Detroit (Kieffer et al. 2005; Thornton et al. 2006). Participating women identified weight, diet, and physical activity beliefs and practices; and individual, family, social and community barriers and facilitators to adopting or maintaining healthy lifestyles during and after pregnancy (Kieffer et al. 2005; Thornton et al. 2006). Many women described feelings of severe social isolation and stress. The absence of mothers and other female relatives and friends to provide social support were prominent barriers to women's ability to maintain healthy practices during and after 
pregnancy (Kieffer et al. 2002; Thornton et al. 2006). Pregnant and postpartum Latinas recommended a group program that emphasized bringing women together to share, support, and learn from each other about healthy eating and exercise (Kieffer et al. 2005). They recommended that the group program be led by women "like them", with shared language, cultural and experiential characteristics. In response, the Healthy MOMs steering committee placed social support at the heart of its theoretical intervention model.

The Healthy MOMs curriculum and activities were tailored to reflect the beliefs and practices discovered during the formative research process, and to respect the language, culture and community context of participating women. While reducing depressive symptoms was not an original study objective, reducing social isolation was central to achieving Healthy MOMs behavioral, recruitment and retention objectives. The intervention design integrated social support from peers during group discussion and intervention activities, and from trained community health workers who facilitated group and one-on-one meetings.

In the current study, we tested the hypothesis that the Healthy MOMs healthy lifestyle intervention reduced depressive symptoms in a general population of Latinas during pregnancy and the early postpartum period.

\section{Methods}

Study Setting, Participant Recruitment, Eligibility and Randomization

The Healthy MOMs study was conducted between 2004 and 2006, in southwest Detroit, a mixed ethnic community, whose Latino population was, and is, predominantly of Mexican/Mexican-American origin, and low income (US Census Bureau 2000). While always the home to Detroit's Latino population, southwest Detroit's immigrant community has grown rapidly in the past 15 years (US Census Bureau 2000, 2009). The rest of the city of Detroit is predominantly African-American (US Census Bureau 2000).

Healthy MOMs was conducted in several community partner organization settings, including Community Health and Social Services (CHASS) Center, a federally qualified health center and trusted community institution that has served the community with comprehensive health and social services since 1970. CHASS was represented on the steering committee, was a major recruitment site and housed data collection activities. Separate community organizations housed intervention and control group meetings. Pregnant women were recruited at CHASS, Supplemental Nutrition Program for Women, Infants, and
Children (WIC) clinics, and through posters and flyers distributed to community organizations and in public settings in Southwest Detroit. A pregnant Latina was eligible to participate in this study if she was $\geq 18$ years of age, a resident of Southwest Detroit, and $<20$ weeks gestational at the eligibility screening.

Eligible women received an orientation session during which community health workers (Women's Health Advocates [WHAs]) and study research staff explained the purpose of the study, the meaning of randomization, the program content and expectations for participants in the intervention and control groups, and data collection procedures. Information about childcare, transportation, and incentives was provided. Informed primary and medical record consent were obtained at this session. Consenting women were then scheduled for three baseline data collection visits. At the end of the third visit, each woman received an incentive payment and a sealed envelope containing her intervention group assignment. These envelopes were prepared in advance by the statistician who generated the random allocation sequence, using a uniform distribution in blocks of 40 for each cohort. Women were equally likely to be randomized to the Healthy MOMs Healthy Lifestyle Intervention group (MOMs) or the Healthy Pregnancy Education (control) group. The study protocol was approved by the Institutional Review Board at the University of Michigan prior to the start of recruitment.

\section{Healthy Lifestyle Group and Control Group} Interventions

Table 1 summarizes the structure and content of MOMs and control group intervention meetings. The MOMs intervention was led by the WHAs, Spanish-speaking, Latina community residents who received extensive training prior to beginning recruitment. The MOMs intervention was offered in a 14-session curriculum conducted weekly in Spanish during two home visits and nine group meetings during pregnancy; and two home visits and one group meeting conducted between 2 and 6 weeks postpartum. Intervention women attended an average of 10.5 sessions (group meetings plus home visits), with $98.6 \%$ attending at least 1 session and $10.1 \%$ attending all 14 sessions. Both group meetings and home visits integrated information, discussion and activities aimed at empowering women to develop knowledge and skills to reduce social and environmental barriers to healthy eating and regular exercise. For example, meeting 4 included discussion of the benefits of physical activity, how to exercise safely during pregnancy, women's perceptions of challenges they face and ideas for including physical activity in their daily lives. Each group meeting concluded with content review and goal setting. Optional weekly group healthy eating and 
Table 1 Comparison of the healthy mothers on the move (MOMs) and control group curricula (all classes are group sessions unless otherwise noted)

\begin{tabular}{llll}
\hline $\begin{array}{l}\text { Class } \\
\text { number }\end{array}$ & Class name & $\begin{array}{l}\text { Healthy } \\
\text { MOMs } \\
\text { curriculum }^{\mathrm{b}}\end{array}$ & $\begin{array}{l}\text { Control } \\
\text { curriculum }\end{array}$ \\
\hline
\end{tabular}

\section{Pregnancy}

\begin{tabular}{llll}
1 & Healthy mom, healthy baby! & $\mathrm{X}$ & $\mathrm{X}$ \\
2 & Plan to be active! & $\mathrm{X}^{\mathrm{a}}$ & \\
3 & Plan to eat healthy! & $\mathrm{X}^{\mathrm{a}}$ & \\
4 & Move more, sit less! & $\mathrm{X}$ & \\
5 & Eat more fiber! & $\mathrm{X}$ & \\
6 & Eat more fruits and vegetables! & $\mathrm{X}$ & \\
7 & Eat less fat and sugar! & $\mathrm{X}$ & \\
8 & Getting ready: labor and birth! & $\mathrm{X}$ & $\mathrm{X}$ \\
9 & Stay motivated! & $\mathrm{X}$ & \\
10 & Healthy activities together! & $\mathrm{X}$ & \\
11 & Infant care! & $\mathrm{X}$ & $\mathrm{X}$ \\
Postpartum & & \\
12 & Mom and baby! & $\mathrm{X}^{\mathrm{a}}$ & $\mathrm{X}$ \\
13 & Health for life! & $\mathrm{X}^{\mathrm{a}}$ & \\
14 & Celebrate success! & $\mathrm{X}$ & \\
\hline
\end{tabular}

${ }^{a}$ Indicates a home visit

b Each MOMs meeting during pregnancy had a corresponding activity day

exercise activities, such as healthy food/cooking demonstrations, walking groups, or aerobic dancing, corresponded to the intervention topic of the week. MOMs participants attended an average of 5.1 activity days with $97.1 \%$ attending at least 1 activity day and $2.2 \%$ attending all activity days.

Social support from the WHAs and peers was a key component of the MOMs intervention. For example, WHA's provided informational support while delivering the intervention curriculum. Home visits were similar in curricular content to group meetings but WHA's also encouraged women to develop and review behavioral goals, and provided emotional support by recognizing their efforts and challenges. WHA's facilitated group discussions of barriers to healthy lifestyles faced by participants, and encouraged women to problem solve and share strategies. Peer support was strengthened by informal conversations about their lives and shared experiences during classes and activity days.

The control group received its intervention during four group meetings; three during pregnancy and one at approximately 6 weeks postpartum. It was delivered by trained staff from a Healthy MOMs partner, a community mental health agency. The content of the control group meetings corresponded with MOMs meetings $1,8,11$ and 12. Control women attended an average of 1.64 sessions (group meetings plus home visit) with $86.1 \%$ attending at least 1 session and $1.5 \%$ attending all sessions. Control group participants also received standard pregnancy education materials about eating and exercise from the March of Dimes and the American College of Obstetricians and Gynecologists.

Both the MOMs and the control groups' curricula included the same educational content regarding pregnancy, childbirth and the postpartum period, and identical content and activities related to identifying and managing stress and identifying signs and symptoms of depression during and after pregnancy during meetings 1 and 12 . Participants in both groups also received "The Little Pregnancy Book", which reviewed maternal and fetal/ newborn development and care; and monthly newsletters with health tips, reminder cards, and phone call reminders for meetings and data collection visits. Following each intervention meeting, participants in both groups received the same small gift incentives related to mother and baby care, such as skin cream, candles, water bottles, and baby bibs. Celebratory graduation ceremonies were held for all participants following completion of study activities. Transportation and child care were provided for all study activities. MOMs and control group meetings were conducted at separate community organizations.

Standardized checklists were completed by a trained observer, intervention facilitators and participants for all group classes and, on a random basis, during home visits. These checklists were used during regular meetings between research staff and intervention facilitators to review fidelity to the curriculum protocol. For our process evaluation, fidelity to each curriculum topic and activity was assessed on a four-point scale from "not covered" (1) to "completely covered" (4). The overall average observer rating (all classes, all cohorts) was 3.82 .

\section{Data Collection}

Data were collected at three time points: pre-intervention (baseline), immediately after the intervention during pregnancy (follow-up), and approximately 6 weeks postpartum. Each time point consisted of three data collection visits separated by approximately 1 week to reduce participant burden. The mean gestational age at the first baseline data collection visit was 17.3 weeks (range 7-27 weeks). The mean gestational age at follow-up was 27.3 weeks (range 16-36 weeks). Postpartum data were collected a mean of 6.9 weeks after delivery (range 2-14 weeks). Data collectors were blinded to study assignment and were employed by a separate institution. They had no involvement with study administration or delivery of the MOMs or control curricula. Data collection was conducted at CHASS and at participants' homes. No 
data collection was conducted in the settings of intervention activities.

\section{Measures}

Depressive symptoms within the past week were measured with the Center for Epidemiologic Studies-Depression Scale (CES-D). This scale is not intended to diagnose depression, but suggests the need for further evaluation. The original 20-item CES-D (Radloff 1977) has a high Cronbach's alpha $(\alpha=0.85)$ and four subscales: depressed affect, positive affect, somatic and retarded activity, and interpersonal. A score of $\geq 16$ indicated that a woman had a "clinically significant level of depressive symptoms" (Orr et al. 2007; Mora et al. 2009). For simplicity, this analysis will consider women who score 16 or over to be "at risk for depression". A shorter (11-item) version of the CES-D was later developed to decrease respondent burden (Kohout et al. 1993; White et al. 1986). This shorter version, which was used in Healthy MOMs, was validated against the 20-item CES-D, had a similar reliability ( $\alpha=0.76$ ) compared to the 20 -item CES-D $(\alpha=0.86)$, and tested the same four factors as the 20-item CES-D (Kohout et al. 1993). Several studies support the use of shortened versions of the CES-D in Mexican immigrant populations (Grzywacz et al. 2006; Perreira et al. 2005; Roberts and Sobhan 1992).

The score on the 11-item CES-D was transformed to the 20-item CES-D score using the method presented in Kohout et al. (Kohout et al. 1993). Briefly, the five response options (never, hardly ever, sometimes, often, always) were collapsed into three categories (never/hardly ever, sometimes, often/always) and the resulting categories were summed to match the scoring described by White et al. (never/hardly ever $=0$, sometimes $=1$, and often/ always $=2$ ) (White et al. 1986). The total score was then transformed to a 20-item CES-D score using a linear regression equation. Using a cut-point of 16 corresponded to the 80th percentile in women when the 20-item CES-D was used (Radloff 1977), and the 78th percentile in women when the transformed 11-item CES-D was used (Kohout et al. 1993). If a woman did not respond to $\geq 6$ of the 11 questions, the CES-D score was not calculated. The Cronbach's alpha for the CES-D scale for Healthy MOMs participants was 0.78 at baseline, 0.82 at follow-up, and 0.85 at postpartum. All 275 women successfully completed the CES-D during at least one of the three time points (baseline, follow-up, postpartum).

\section{Statistical Analysis}

Maternal characteristics of the MOMs and control groups were compared using Pearson's $\chi^{2}$ test or Fisher's exact test, as appropriate.

\section{Continuous CES-D Score}

The primary analysis was an unadjusted comparison using linear mixed models to allow for correlations among observations on the same woman. These models were used to estimate the mean CES-D score with $95 \%$ confidence intervals (CIs) and to make comparisons between and within the MOMs intervention and control groups at the baseline, follow-up and postpartum time points. Using this methodology allows us to conduct an "intention-to-treat" analysis, as is standard practice when analyzing randomized controlled trials (Hollis and Campbell 1999). Intention-to-treat analysis includes all participants in the analysis, regardless of the number of classes attended or the number of follow-up data collection visits completed. This approach allows investigators to maintain between group randomization with respect to participant characteristics and to allow for participant non-compliance, as might occur in routine clinical practice (Hollis and Campbell 1999). Thus, if a woman had data for the CES-D for at least one study time point, she was included in this analysis. The final sample size of the primary (unadjusted) analysis was 275 women (MOMs, $\mathrm{n}=138$; control, $\mathrm{n}=137$ ).

A secondary analysis was conducted adjusting the linear mixed model for covariates considered theoretically important for the analysis: age at baseline interview (centered at the median: 27 years), years lived in the United States (categories: $<2$ [ref], 2-5, 6-9, $\geq 10$ years), married and living with spouse (yes [ref] vs. no), prenatal care received at CHASS (yes vs. no [ref]), and parity (0 [ref] vs. $\geq 1$ ). This model also included the only variable that differed between randomization groups at baseline (English speaking ability: none vs. other [ref]). The final sample size for the secondary (adjusted) analysis was 263 women (MOMs, $\mathrm{n}=136$; control, $\mathrm{n}=127$ ).

A sub-group analysis was used to estimate the intervention effect among women who did not speak any English at baseline. This sub-group comprised a majority $(82.8 \%)$ of the population analyzed in the secondary (adjusted) analysis. Both unadjusted and adjusted models were considered in this sub-group analysis. The final sample size for this sub-group analysis was 218 women (MOMs, $\mathrm{n}=117$; control, $\mathrm{n}=101$ ).

\section{Categorical CES-D Score}

Generalized linear models with generalized estimating equations (GEE) to allow for correlation among multiple observations on the same person were used to analyze the categorical outcome of being at risk for depression (CES$\mathrm{D} \geq 16$ ). This analysis compared the odds of being at risk for depression at follow-up or at postpartum for women in the MOMs group compared to women in the control group. 
A total of 249 women were included in this analysis (MOMs $\mathrm{n}=125$, control $\mathrm{n}=124)$. An adjusted analysis was also carried out for the categorical outcome that adjusted for the following covariates: baseline depression category (CES-D: $<16$ vs. $\geq 16$ ), covariates considered theoretically important, as described above, and English-speaking ability. Only the 238 women (MOMs $\mathrm{n}=123$; control $\mathrm{n}=115$ ) who completed the CES-D at baseline and at least one additional time point (follow-up and/or postpartum) and who had complete covariate data were included in this adjusted analysis. Effect sizes were calculated for the difference in proportions between the MOMs and control groups using Cohen's h with the arcsine transformation (Cohen 1988). Interpretation of Cohen's h is comparable to Cohen's d, with effect sizes of small $=\leq 0.2$, medium $=0.5$ and large $=0.8 \quad($ Cohen 1988).

The data analysis for this paper was conducted using SAS software, Version 9.2 of the SAS System for Windows (SAS Institute Inc., Cary, NC).

\section{Results}

Eligibility screening for Healthy MOMs began in January 2004 and the final series of intervention classes ended in October 2006. Figure 1 illustrates the flow of participants through the study. Although 278 women were randomized, three of these women were excluded from all analyses after they were later found to be ineligible: in the MOMs group, one woman incorrectly reported the date of her last normal menstrual period by 15 weeks, entering the study at 35 weeks gestation; in the control group, one woman did not complete the baseline data collection visit and another woman delivered twins. All women were analyzed according to their original group assignment, in accordance with the intention-to-treat analysis approach (Hollis and Campbell 1999). CES-D scores were available for 199 women $(72.4 \%)$ at all three time points, 49 women $(17.8 \%)$ at two time points and 27 women $(9.8 \%)$ at one time point.
Fig. 1 Flow of participants through healthy mothers on the move (healthy MOMs) study

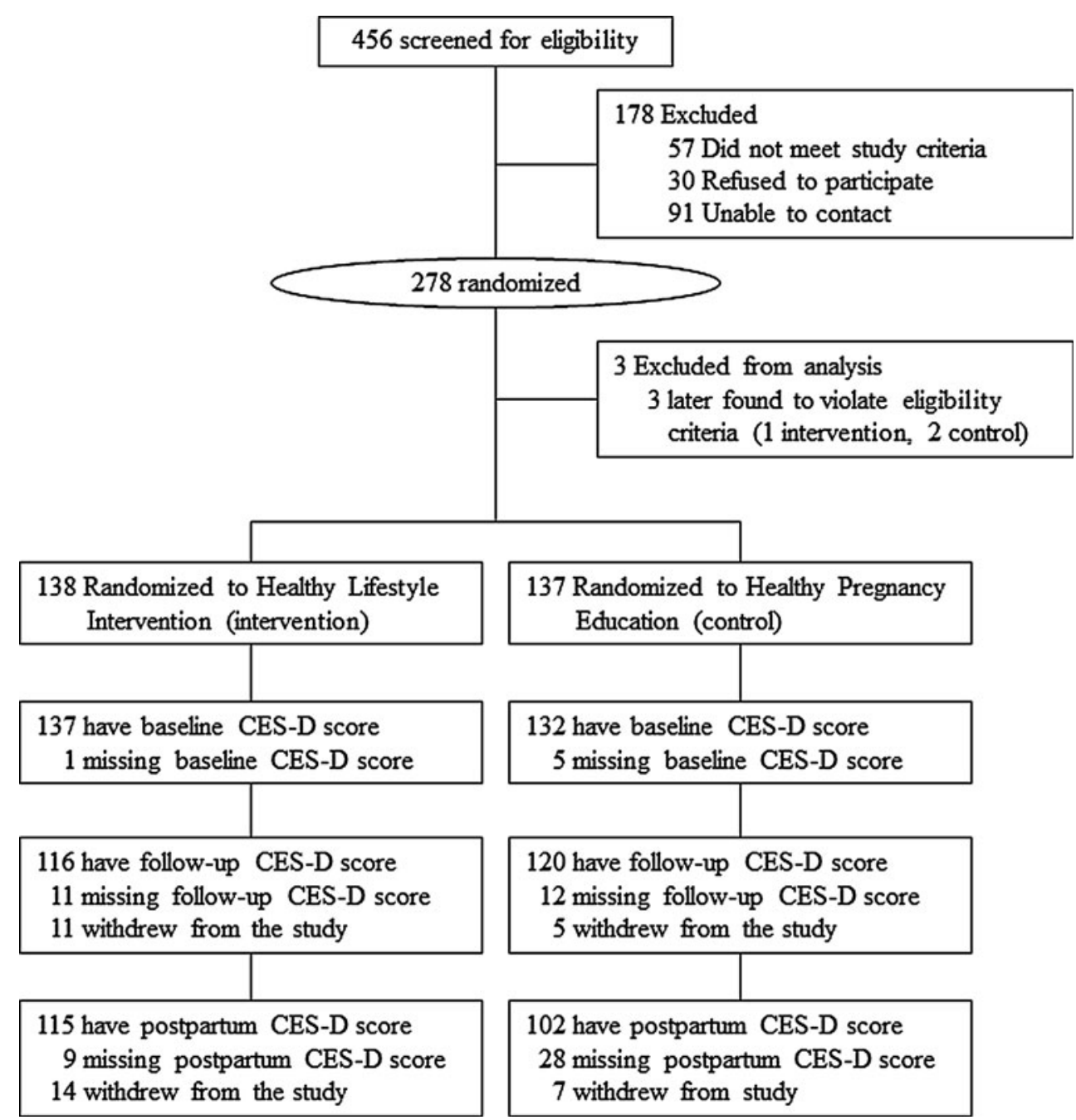


Approximately $90 \%$ of all participants were born in Mexico and considered themselves to be of Mexican/Chicano ethnicity (Table 2). More than half of all study participants were married $(58.6 \%)$ and nearly all were homemakers $(90.3 \%)$. A larger proportion of women in the MOMs group compared to the control group did not speak any English $(p=0.040)$. There were no other significant differences between the MOMs and control women for other characteristics. The baseline CES-D score was

Table 2 Baseline characteristics of study participants $(n=275)$ : healthy mothers on the move (healthy MOMs) study, Detroit, Michigan

\begin{tabular}{|c|c|c|c|c|c|}
\hline & \multicolumn{2}{|c|}{$\begin{array}{l}\text { MOMs } \\
(\mathrm{n}=138)\end{array}$} & \multicolumn{2}{|c|}{$\begin{array}{l}\text { Control } \\
\text { group } \\
(\mathrm{n}=137)\end{array}$} & \multirow[t]{2}{*}{$p$ Value $^{\mathrm{a}}$} \\
\hline & $\mathrm{N}$ & $\%$ & $\mathrm{n}$ & $\%$ & \\
\hline Age, years & & & & & 0.95 \\
\hline $18-24$ & 48 & 34.8 & 47 & 34.3 & \\
\hline $25-29$ & 42 & 30.4 & 44 & 32.2 & \\
\hline$\geq 30$ & 48 & 34.8 & 46 & 33.6 & \\
\hline Education, years & & & & & 0.51 \\
\hline$\leq 6$ & 43 & 31.2 & 35 & 25.9 & \\
\hline $7-11$ & 53 & 38.4 & 51 & 37.8 & \\
\hline$\geq 12$ & 42 & 30.4 & 49 & 36.3 & \\
\hline Birthplace & & & & & 0.17 \\
\hline Mexico & 128 & 92.8 & 116 & 85.9 & \\
\hline Mainland United States & 3 & 2.2 & 5 & 3.7 & \\
\hline Other & 7 & 5.1 & 14 & 10.4 & \\
\hline Years lived in United States & & & & & 0.39 \\
\hline$<2$ & 18 & 13.1 & 18 & 13.7 & \\
\hline $2-5$ & 54 & 39.4 & 51 & 38.9 & \\
\hline $6-9$ & 31 & 22.6 & 39 & 29.8 & \\
\hline$\geq 10$ & 34 & 24.8 & 23 & 17.6 & \\
\hline Does not speak English at all & 117 & 84.8 & 101 & 74.8 & 0.040 \\
\hline Married and living with spouse & 83 & 60.6 & 74 & 56.5 & 0.50 \\
\hline Occupation: homemaker & 124 & 90.5 & 118 & 90.1 & 0.90 \\
\hline Parity & & & & & 0.34 \\
\hline 0 & 38 & 27.5 & 29 & 21.5 & \\
\hline $1-2$ & 78 & 56.5 & 88 & 65.2 & \\
\hline$\geq 3$ & 22 & 15.9 & 18 & 13.3 & \\
\hline $\begin{array}{l}\text { Received prenatal care at } \\
\text { CHASS }^{\mathrm{b}}\end{array}$ & 92 & 66.7 & 95 & 69.3 & 0.63 \\
\hline $\begin{array}{l}\text { At risk for depression } \\
\left(\mathrm{CES}-\mathrm{D}^{\mathrm{c}} \geq 16\right)\end{array}$ & 54 & 39.4 & 44 & 33.3 & 0.30 \\
\hline
\end{tabular}

Numbers may not add to total due to missing data

${ }^{a} p$ Values were obtained with the Fisher's exact test for variables where the expected cell count was $<5$ and the Pearson $\chi^{2}$ test for all other categorical variables

b Three or more prenatal visits at Community Health and Social Services (CHASS) Center

${ }^{c}$ Center for Epidemiologic Studies - Depression Scale $\geq 16$ for $39.4 \%$ of women in the MOMs group and $33.3 \%$ of women in the control group $(p=0.30)$, indicating that approximately one-third of study participants were at an increased risk for depression at baseline.

\section{Continuous CES-D Score}

In the unadjusted analysis, the mean CES-D scores of the MOMs women at baseline, follow-up and postpartum were 13.23 points, 11.24 points, and 10.56 points, respectively (Table 3). The mean CES-D score for MOMs women decreased significantly between baseline and follow-up $(p=0.002)$, but the decrease between follow-up and postpartum was not significant $(p=0.30)$. The overall decrease in mean CES-D score from baseline to postpartum was highly significant for the MOMs group $(p<0.001)$. The mean CES-D scores of control group women at baseline, follow-up and postpartum were 12.87 points, 12.71 points, and 11.65 points, respectively. The mean CES-D score for control women did not significantly decrease between baseline and follow-up $(p=0.79)$ or between follow-up and postpartum $(p=0.12)$. However, the overall decreased in mean CES-D score from baseline to postpartum was marginally significant $(p=0.068)$.

The intervention effect was estimated using post hoc contrasts to compare the change from baseline to follow-up and from baseline to postpartum for the MOMs versus the control group. The MOMs group had a significantly greater decrease in CES-D score from baseline to follow-up than the control group (mean difference in change score $=$ -1.83 points; $95 \%$ CI: $-3.59,-0.07 ; p=0.042$ ). Overall, from baseline to postpartum, the mean CES-D score of the MOMs group decreased 1.45 points more than the mean CES-D score of the control group, although this difference in overall change scores was not significant (95\% CI: $-3.26,0.37 ; p=0.12$ ). After adjusting for additional participant characteristics, the overall intervention effect was marginally significant (mean difference in change score $=-1.62 ; 95 \%$ CI: $-3.47,0.24 ; p=0.087$ ) (Table 3).

To estimate the effect of the intervention with respect to acculturation, a sub-analysis was carried out among women who did not speak any English at baseline $(\mathrm{n}=218)$. Due to the small number of women who spoke at least some English at baseline, a separate analysis was not carried out among this group $(n=55)$. The mean CES-D score for MOMs women who did not speak any English decreased 1.75 points between baseline and follow-up $(p=0.008)$ and then decreased an additional 0.78 points between follow-up and postpartum $(p=0.25)$. The mean CES-D score for control women decreased 0.66 points between baseline and follow-up $(p=0.36)$ and then increased 0.21 points between follow-up and postpartum $(p=0.78)$. Although 
Table 3 Linear mixed model estimates of: mean CES-D scores at baseline (mean 17.3 weeks gestation), follow-up (mean 28.0 weeks gestation), and postpartum (mean 8.3 weeks postpartum); the change from baseline to each follow-up time point within each group; and the statistical significance of the intervention effect between baseline and each follow-up time point

\begin{tabular}{|c|c|c|c|c|c|c|}
\hline & \multirow{2}{*}{$\begin{array}{l}\text { Baseline } \\
\text { Mean }\left(\mathrm{SE}^{\mathrm{a}}\right)\end{array}$} & \multirow{2}{*}{$\begin{array}{l}\text { Follow-up } \\
\text { Mean (SE) }\end{array}$} & \multirow{2}{*}{$\begin{array}{l}\text { Postpartum } \\
\text { Mean (SE) }\end{array}$} & \multicolumn{3}{|l|}{ Change from: } \\
\hline & & & & $\begin{array}{l}\text { Baseline to follow-up } \\
\text { Mean (SE) }\end{array}$ & $\begin{array}{l}\text { Follow-up to } \\
\text { postpartum } \\
\text { Mean (SE) }\end{array}$ & $\begin{array}{l}\text { Baseline to } \\
\text { postpartum } \\
\text { Mean (SE) }\end{array}$ \\
\hline \multicolumn{7}{|c|}{ Intent-to-treat analysis ${ }^{\mathrm{b}}$} \\
\hline MOMs & $13.23(0.64)$ & $11.24(0.68)$ & $10.56(0.68)$ & $-1.99(0.63)^{* *}$ & $-0.68(0.66)$ & $-2.67(0.63)^{* * *}$ \\
\hline Control & $12.87(0.65)$ & $12.71(0.67)$ & $11.65(0.70)$ & $-0.17(0.63)$ & $-1.06(0.68)$ & $-1.22(0.67)^{\dagger}$ \\
\hline Intervention effect $^{\mathrm{c}}$ & & & & $-1.83(0.89)^{*}$ & $0.38(0.94)$ & $-1.45(0.92)$ \\
\hline \multicolumn{7}{|l|}{ Adjusted analysis ${ }^{\mathrm{d}}$} \\
\hline MOMs & $12.98(1.52)$ & $10.98(1.53)$ & $10.18(1.53)$ & $-2.00(0.64)^{* *}$ & $-0.80(0.67)$ & $-2.79(0.64)^{* *}$ \\
\hline Control & $12.88(1.56)$ & $12.87(1.58)$ & $11.70(1.59)$ & $-0.01(0.65)$ & $-1.16(0.70)$ & $-1.18(0.69)^{\dagger}$ \\
\hline Intervention effect & & & & $-1.98(0.91)^{*}$ & $0.37(0.97)$ & $-1.62(0.94)^{\dagger}$ \\
\hline
\end{tabular}

Center for Epidemiologic Studies-Depression Scale

$* * * p<0.001$

$* * p<0.01$

$* p<0.05$

${ }^{\dagger} p<0.10$

${ }^{a}$ Standard error of the mean based on the linear mixed model

${ }^{b}$ The intention-to-treat analysis is unadjusted and includes all randomized women $(\mathrm{N}=275$; intervention group $\mathrm{n}=138$; control group $\mathrm{n}=137$ )

${ }^{c}$ Intervention effect $=$ the change in the intervention group for a specific time period minus the change in the control group for the same time period

${ }^{\mathrm{d}}$ Adjusted for age at the baseline interview, years lived in the United States, married \& living with spouse, prenatal care received at Community Health and Social Services (CHASS) Center, and English-speaking ability $(\mathrm{N}=263$; intervention group $\mathrm{n}=136$; control group $\mathrm{n}=127)$

the intervention effects were not significant between baseline and follow-up $(p=0.26)$ and between follow-up and postpartum $(p=0.34)$, the decline in CES-D score from baseline to postpartum was significantly greater for MOMs participants compared to control participants among this sub-group of women who did not speak any English (mean difference in change scores $=-2.08 ; 95 \%$ CI $=-4.08,-0.09 ; p=0.041)$.

\section{Categorical CES-D Score}

Among the 249 participants included in the unadjusted categorical CES-D score analysis, there was no significant difference in the percent of women at risk for depression (CES-D $\geq 16$ ) in the two study groups at baseline $\left(\mathrm{MOMs}=40.3 \%, \quad\right.$ control $=32.8 \% ; \quad \chi^{2}=1.49, \quad p=$ 0.22 ). In the unadjusted GEE analysis, there was no significant difference in the percent of women at risk for depression in the MOMs versus control groups at either the follow-up $(p=0.10)$ or the postpartum time point $(p=0.95)$ (Table 4). After adjusting for baseline depressive symptoms score, the percentage of women at risk for depression at follow-up was significantly less in the MOMs group than the percentage in the control group (19.0 vs.
$33.7 \%, p=0.019$; effect size $=0.34$ ), but this difference was not significant at the postpartum time point (19.8 vs. $22.4 \%, p=0.65$; effect size $=0.06$ ). The GEE analysis that was adjusted for baseline depression and other participant characteristics also showed a significantly lower percentage of women in the MOMs group at risk for depression than in the control group at follow-up (18 vs. $36.3 \%, p=0.005$; effect size $=0.42$ ), but not significantly different in the percentages at the postpartum time point $(18.7$ vs. $23.7 \%, p=0.40$; effect size $=0.12)$.

\section{Discussion}

The results of this study suggest that the Healthy MOMs healthy lifestyle program may offer a promising model for community-based interventions that seek to reduce depressive symptoms among low income, immigrant pregnant and early postpartum Latinas. The effect of the MOMs intervention on depressive symptoms was strongest during pregnancy. Non-English speaking women, who constituted the large majority of participants, may also derive some longer term benefit related to a reduced level of depressive symptoms. This study had several novel 
Table 4 Percent of MOMs and control women at risk for depression (CES-D $\geq 16$ ) and adjusted odds ratios at the follow-up and postpartum time points estimated using GEE models

\begin{tabular}{|c|c|c|c|c|c|c|}
\hline & \multicolumn{3}{|c|}{ Follow-up } & \multicolumn{3}{|c|}{ Postpartum } \\
\hline & \multicolumn{2}{|c|}{ CES-D $\geq 16$} & \multirow[t]{2}{*}{ OR $(95 \% \mathrm{CI})$} & \multicolumn{2}{|c|}{ CES-D $\geq 16$} & \multirow[t]{2}{*}{ OR $(95 \% \mathrm{CI})$} \\
\hline & $\%$ & $95 \% \mathrm{CI}$ & & $\%$ & $95 \% \mathrm{CI}$ & \\
\hline \multicolumn{7}{|c|}{ Unadjusted $(n=249)$} \\
\hline MOMs & 24.4 & $(17.5,33.0)$ & $0.62(0.36,1.09)$ & 25.0 & $(18.0,33.6)$ & $0.98(0.53,1.80)$ \\
\hline Control & 34.2 & $(26.3,43.0)$ & & 25.4 & $(18.0,34.6)$ & \\
\hline \multicolumn{7}{|c|}{ Adjusted for baseline depression category only $(n=243)$} \\
\hline MOMs & 19.0 & $(12.5,27.6)$ & $0.46(0.24,0.88)^{*}$ & 19.8 & $(13.4,28.3)$ & $0.86(0.43,1.69)$ \\
\hline Control & 33.7 & $(24.7,44.2)$ & & 22.4 & $(14.7,32.5)$ & \\
\hline \multicolumn{7}{|c|}{ Adjusted for baseline depression category and additional participant characteristics $(n=238)$} \\
\hline MOMs & 18.0 & $(11.8,26.6)$ & $0.39(0.20,0.75)^{* *}$ & 18.7 & $(12.6,26.9)$ & $0.74(0.37,1.49)$ \\
\hline Control & 36.3 & $(26.5,47.4)$ & & 23.7 & $(15.4,34.8)$ & \\
\hline \multicolumn{7}{|c|}{$* * p<0.01$} \\
\hline \multicolumn{7}{|c|}{$* p<0.05$} \\
\hline
\end{tabular}

aspects. Unlike studies of interventions specifically targeting pregnant or postpartum Latina women with high levels of depressive symptoms or clinical depression (Beeber et al. 2010; Le et al. 2011; Muñoz et al. 2007; Spinelli and Endicott 2003), our study population was not limited to women with, or at high risk for, depression. It represented the range of pregnant women usually seen in obstetric and other primary care settings. Most depression interventions used mental health professionals or research assistants to deliver targeted psychotherapeutic interventions (Dennis and Creedy 2004; Grote et al. 2009; Miranda et al. 2003; O'Hara 2009; Roman et al. 2009). The Healthy MOMs intervention promoted healthy eating and regular exercise during pregnancy and the early postpartum, and was delivered by non-professional women from the same community.

Latinos/as commonly underutilize traditional mental health services and treatments (McGarry et al. 2009; Stacciarini 2008). In a Utah study of women experiencing postpartum depression, Hispanic women were three times less likely than non-Hispanic white women to seek help (McGarry et al. 2009). Latinas may prefer counseling and culturally tailored interventions to traditional antidepressant medications and therapies (Beeber et al. 2010; Givens et al. 2007; McGarry et al. 2009; Stacciarini 2008). Culturally tailored, professionally administered interventions for pregnant and postpartum Latinas with major depression or high levels of depressive symptoms have been developed (Beeber et al. 2010; Le et al. 2011; Spinelli and Endicott 2003). Although our intervention was delivered by community health workers and focused on developing healthy lifestyle habits in Latinas with varying levels of depressive symptoms, our results are generally consistent with other culturally tailored interventions that observed a significant intervention effect on depressive symptoms during pregnancy (Le et al. 2011; Spinelli and Endicott 2003).

Depressive symptoms decreased in both the MOMs and control groups from baseline to postpartum. This is consistent with other studies conducted with Latinas (Diaz et al. 2007; Le et al. 2011; Zayas et al. 2003). However, the pattern of change in CES-D score varied by group in our study. In the control group, depressive symptoms did not decline during pregnancy, from baseline to post-intervention follow-up, but did decrease slightly between follow-up and 6 weeks postpartum. In the MOMs intervention group, depressive symptoms decreased significantly between baseline and follow-up during pregnancy and then remained stable to 6 weeks postpartum. Similar to the findings of Le et al. (Le et al. 2011), the significant intervention effect we observed did not extend into the early postpartum period when the study population was considered overall. Among non-English-speaking MOMs participants, however, depressive symptoms continued to decline into the postpartum period; whereas, there was no decline among non-English-speaking women in the control group. The overall baseline to postpartum intervention effect was significant among non-English-speaking women.

Although Vega et al. described lower levels of depression among immigrant Latinos compared to those born in the US (Vega et al. 2011), immigrant, pregnant Latinas in Detroit are frequently separated from female relatives and 
other community supports that might buffer them from psychosocial stressors that may influence depression (Kieffer et al. 2002). This separation, and a language barrier, enhances the sense of social isolation reported by many Latina immigrants (Heilemann et al. 2004; Kieffer et al. 2002; Martinez-Schallmoser et al. 2003). The integration of social support from peers and the WHA's in the study design may have contributed to successfully reducing depressive symptoms among monolingual Spanish-speaking MOMs participants.

It has been recommended that data from randomized controlled trials be analyzed according to the intention-totreat principle in an unadjusted analysis (Hollis and Campbell 1999). However, in this study adjustment for covariates was important. There were significantly more non-English speaking women in the MOMs group than the control group. While unadjusted results were similar to adjusted results in most analyses, adjusting for covariates led to a marginally significant baseline to postpartum intervention effect when depressive symptoms were considered as a continuous variable. In categorical analyses, adjustment for baseline depression level revealed the significant intervention effect at follow-up during pregnancy.

The participation of Latinas from the same community in Healthy MOMs planning, design and implementation contributed to the cultural acceptability of its curriculum, activities and structure, and the ability of its staff to help address the complex social and environmental factors that may contribute to depression during and after pregnancy. Trusted community-based settings, child care and transportation helped to reduce common barriers to participation among low income women (Kieffer et al. 2002; Miranda et al. 2003). Although Healthy MOMs was not based on a CBT model, some of its structural elements, such as group education, skill building and problem solving were present, and are considered adaptive to Latino cultural values (Le et al. 2011; Muñoz et al. 2007). These aspects of the Healthy MOMs intervention may have helped participants manage the feelings that arise from relatively uncontrollable aspects of their daily lives (Le et al. 2011; Muñoz et al. 2007). Nonetheless, issues such as family instability, violence, ill health, pregnancy and newborn health complications, and major depression, are likely to require the addition of more intensive and personalized forms of therapy at least in complement to an intervention such as Healthy MOMs (Spinelli and Endicott 2003).

Reviews of depression interventions for postpartum women have suggested a lack of evidence for the efficacy of interventions that target all women in the population versus those identified at high risk (O'Hara 2009) and stronger evidence for the efficacy of individualized (versus group) interventions conducted by professionals (Dennis and Creedy 2004). However, these studies were largely conducted outside of the United States, and not with Latina populations. The results of our study suggest that a communitybased healthy lifestyle intervention can successfully reach pregnant and early postpartum Spanish-speaking Latinas and reduce depressive symptoms among them while promoting healthy eating and regular exercise. Similar interventions, based on community-based participatory research approaches during the formative stage, may hold promise for reducing depressive symptoms during pregnancy among Latinas in other communities and primary care settings. The MOMs intervention, which also provides pregnancy education and promotes maternal health and chronic disease prevention may be especially relevant in the context of current health care reform efforts that include incentives to integrate mental health screening and treatment in primary care, and to address lifestyle and medical risk in behavioral health care (Collins et al. 2010; Substance Abuse and Mental Health Services Administration (SAMHSA) 2010). Future studies are needed to replicate these findings and to understand characteristics of women who benefited the most from the intervention. Formative research is needed to assess if, and how, the Healthy MOMs intervention might be adapted to address the needs of women with major depression without dissuading women from participating because of the stigma attached to identifying oneself, or being identified, as depressed.

There are several limitations to our study. Because the MOMs intervention was not designed to diagnose or treat depression, a measure of clinical depression (e.g. Diagnostic Interview Schedule [DIS]) was not included at any of the three data collection points. Therefore, we cannot directly evaluate the clinical significance of our depressive symptoms findings. In the original validation study of the CES-D depression screening instrument by Radloff et al., a cut-off score of 16 distinguished between psychiatric inpatient and general population samples (Radloff 1977). Recent studies suggest using cut-points of 16 or higher for clinically significant levels of depressive symptoms, or 23 or higher to identify major depressive disorder (Mora et al. 2009; Orr et al. 2007). Because of their immigrant status and potential social isolation, we used the 16 point cut-off to indicate women who were at risk for depression. Although modest, the effect size for the intervention suggests that after adjusting for baseline level of depressive symptoms, the MOMs intervention reduced the number of participants with high levels of depressive symptoms by half when compared to control participants.

We could not address the impact of the MOMs intervention on the reduction of depressive symptoms among US-born Latinas due to the very small number of such participants. The risk of depression is frequently more prevalent in this population and also increases with years of acculturation (Davila et al. 2009; Fortner et al. 2011; 
Heilemann et al. 2004). Future studies are needed to assess whether interventions such as Healthy MOMs or Mamás y Bebés (Le et al. 2011; Muñoz et al. 2007) have similar results among US born Latinas or women with greater levels of acculturation.

The intention-to-treat study design specifically does not account for number of participant contacts in assessing the impact of the intervention on depressive symptoms. Retention contacts were received in equal numbers by participants in both study arms. However, the greater number of intervention meetings and concurrent receipt of a greater number of small gift incentives related to each meeting could have contributed to greater participation and engagement by MOMs compared to control group participants. The positive benefits of exercise and nutrition resulting from participation could also have contributed to the intervention effect on depressive symptoms. However, social support was an integral component of all of our healthy eating and exercise activities. Therefore, the significance of social support to a group of immigrant women who tend to be socially isolated cannot be ignored.

Logistical barriers to participation in depression interventions are common for pregnant and, especially, postpartum women. These include family and other work responsibilities, lack of child care and transportation, and other access barriers (O'Hara 2009). These may be exacerbated for immigrant Latinas who are living in isolated, low income communities with few or no family supports (Kieffer et al. 2002; Thornton et al. 2006). While our study demonstrated that an intervention conducted in trusted community settings can overcome many of these barriers, grant funds supported the transportation and on-site childcare that made participation feasible for our participants. Such supports may not be available in many clinic or community settings. Similarly, although community health workers are gaining increasing attention for their success in improving health care access, quality and outcomes (Felix et al. 2011; Roman et al. 2009; Spencer et al. 2011), most health systems are not yet employing them except through short term grant mechanisms, and most health insurers are not yet providing reimbursement for their services. This study lends additional support to calls for integrating community health workers into public health and medical care services and assuring their support through adequate financing and reimbursement strategies (Brownstein et al. 2011; Spencer et al. 2011).

\section{Conclusion}

Healthy MOMs was a culturally tailored, Spanish-language, community-based healthy lifestyle intervention that provided social support from peers and trained community health workers. It achieved high levels of participation and reduced depressive symptoms in a population that often shies away from, or finds it difficult to participate in traditional mental health services (McGarry et al. 2009; Miranda et al. 2003). Both the MOMs group and the control group participated in general discussions of pregnancy and postpartum physical and emotional changes, recognition of signs and symptoms of depression and stress management techniques. When combined with the health promoting activities and social support provided by the MOMs intervention, the results of this study suggest that a healthy lifestyle intervention may be well-suited to assist in preventing or reducing depressive symptoms among immigrant Latinas during pregnancy, without the stigma often attached to mental health treatment in the Latino community.

Acknowledgments The authors wish to thank Emily Vogtmann and Dr. Erin Rees Clayton for their preliminary examination of the data and the staff at Community Health and Social Services (CHASS) Center and the REACH Detroit Partnership for their guidance and support in developing and conducting these studies. This work was supported by the National Institute of Diabetes and Digestive and Kidney Diseases at the National Institutes of Health (R18 DK062433); the Biostatistics and Measurement Cores of the Michigan Diabetes Research and Training Center (National Institute of Diabetes and Digestive and Kidney Diseases, P60 DK020572; The Centers for Disease Control and Prevention, Division of Nutrition and Physical Activity (U48/CCUS1577S-/SIP 10) the Maternal and Child Health Bureau, Health Resources and Services Administration (R40 MC00115-03) and the University of Michigan Vivian A. and James L. Curtis School of Social Work Research and Training Center.

\section{References}

Beeber, L. S., Holditch-Davis, D., Perreira, K., Schwartz, T. A., Lewis, V., Blanchard, H., et al. (2010). Short-term in-home intervention reduces depressive symptoms in Early Head Start Latina mothers of infants and toddlers. Research in Nursing \& Health, 33(1), 60-76.

Brownstein, J. N., Hirsch, G. R., Rosenthal, E. L., \& Rush, C. H. (2011). Community health workers " 101 " for primary care providers and other stakeholders in health care systems. The Journal of Ambulatory Care Management, 34(3), 210-220.

Cohen, J. (1988). Differences between proportions. In: Cohen, J. (Eds.), Statistical power analysis for the behavioral sciences (2nd Edn., pp. 179-213). Hillsdale, NJ: Lawrence Erlbaum Associates.

Collins, C., Hewson, D. L., Munger, R., \& Wade, T. (2010). Evolving models of behavioral health integration in primary care. New York, NY: Milbank Memorial Fund.

Davila, M., McFall, S. L., \& Cheng, D. (2009). Acculturation and depressive symptoms among pregnant and postpartum Latinas. Maternal and Child Health Journal, 13(3), 318-325.

Dennis, C. L., \& Creedy, D. (2004). Psychosocial and psychological interventions for preventing postpartum depression. The Cochrane Database of Systematic Reviews, 4, CD001134.

Diaz, M. A., Le, H. N., Cooper, B. A., \& Muñoz, R. F. (2007). Interpersonal factors and perinatal depressive symptomatology in a low-income Latina sample. Cultural Diversity \& Ethnic Minority Psychology, 13(4), 328-336. 
Diego, M. A., Field, T., Hernandez-Reif, M., Schanberg, S., Kuhn, C., \& Gonzalez-Quintero, V. H. (2009). Prenatal depression restricts fetal growth. Early Human Development, 85(1), 65-70.

Felix, H. C., Mays, G. P., Stewart, M. K., Cottoms, N., \& Olson, M. (2011). Medicaid savings resulted when community health workers matched those with needs to home and community care. Health Affairs, 30(7), 1366-1374.

Flynn, H. A., Henshaw, E., O’Mahen, H., \& Forman, J. (2010). Patient perspectives on improving the depression referral processes in obstetrics settings: A qualitative study. General Hospital Psychiatry, 32(1), 9-16.

Fortner, R. T., Pekow, P., Dole, N., Markenson, G., \& Chasan-Taber, L. (2011). Risk factors for prenatal depressive symptoms among Hispanic women. Maternal and Child Health Journal, 15(8), $1287-1295$.

Gavin, N. I., Gaynes, B. N., Lohr, K. N., Meltzer-Brody, S., Gartlehner, G., \& Swinson, T. (2005). Perinatal depression: A systematic review of prevalence and incidence. Obstetrics and Gynecology, 106(5 Pt 1), 1071-1083.

Gavin, A. R., Holzman, C., Siefert, K., \& Tian, Y. (2009). Maternal depressive symptoms, depression, and psychiatric medication use in relation to risk of preterm delivery. Women's Health Issues, 19(5), 325-334.

Givens, J. L., Houston, T. K., Van Voorhees, B. W., Ford, D. E., \& Cooper, L. A. (2007). Ethnicity and preferences for depression treatment. General Hospital Psychiatry, 29(3), 182-191.

Grote, N. K., Bridge, J. A., Gavin, A. R., Melville, J. L., Iyengar, S., \& Katon, W. J. (2010). A meta-analysis of depression during pregnancy and the risk of preterm birth, low birth weight, and intrauterine growth restriction. Archives of General Psychiatry, 67(10), 1012-1024.

Grote, N. K., Swartz, H. A., Geibel, S. L., Zuckoff, A., Houck, P. R., \& Frank, E. (2009). A randomized controlled trial of culturally relevant, brief interpersonal psychotherapy for perinatal depression. Psychiatric Services, 60(3), 313-321.

Grzywacz, G. J., Hovey, J. D., Seligman, L. D., Arcury, T. A., \& Quandt, S. A. (2006). Evaluating short-form versions of the CES-D for measuring depressive symptoms among immigrants from Mexico. Hispanic Journal of Behavioral Sciences, 28(3), 404-424.

Heilemann, M., Frutos, L., Lee, K., \& Kury, F. S. (2004). Protective strength factors, resources, and risks in relation to depressive symptoms among childbearing women of Mexican descent. Health Care for Women International, 25(1), 88-106.

Hollis, S., \& Campbell, F. (1999). What is meant by intention to treat analysis? Survey of published randomised controlled trials. British Medical Journal, 319(7211), 670-674.

Hromi-Fiedler, A., Bermúdez-Millán, A., Segura-Pérez, S., \& PérezEscamilla, R. (2011). Household food insecurity is associated with depressive symptoms among low-income pregnant Latinas. Maternal and Child Health Journal, 7(4), 421-430.

Israel, B. A., Lichtenstein, R., Lantz, P., McGranaghan, R., Allen, A., Guzmán, J. R., et al. (2001). The detroit community-academic Urban Research Center: Development, implementation, and evaluation. Journal of Public Health Management and Practice, 7(5), 1-19.

Jesse, D. E., \& Swanson, M. S. (2007). Risks and resources associated with antepartum risk for depression among rural southern women. Nursing Research, 56(6), 378-386.

Kieffer, E. C., Salabarría-Peña, Y., Odoms-Young, A. M., Willis, S. K., Baber, K. E., \& Guzmán, J. R. (2005). The application of focus group methodologies to community-based participatory research. In B. A. Israel, E. Eng, A. J. Schulz, \& E. A. Parker (Eds.), Methods for Conducting Community-Based Participatory Research in Public Health (pp. 146-166). San Francisco, CA: Jossey-Bass.
Kieffer, E. C., Willis, S. K., Arellano, N., \& Guzmán, R. (2002). Perspectives of pregnant and postpartum Latino women on diabetes, physical activity, and health. Health Education \& Behavior, 29(5), 542-556.

Kieffer, E. C., Willis, S. K., Odoms-Young, A. M., Guzmán, J. R., Allen, A. J., Two Feathers, J., et al. (2004). Reducing disparities in diabetes among African American and Latino residents of Detroit: The essential role of community planning focus groups. Ethnicity and Disease, 14(3 Suppl 1), S27-S37.

Kohout, F. J., Berkman, L. F., Evans, D. A., \& Cornoni-Huntley, J. (1993). Two shorter forms of the CES-D (Center for Epidemiological Studies Depression) depression symptoms index. Journal of Aging \& Health, 5(2), 179-193.

Kuo, W. H., Wilson, T. E., Holman, S., Fuentes-Afflick, E., O'Sullivan, M. J., \& Minkoff, H. (2004). Depressive symptoms in the immediate postpartum period among Hispanic women in three US cities. Journal of Immigrant Health, 6(4), 145-153.

Lancaster, C. A., Gold, K. J., Flynn, H. A., Yoo, H., Marcus, S. M., \& Davis, M. M. (2010). Risk factors for depressive symptoms during pregnancy: A systematic review. American Journal of Obstetrics and Gynecology, 202(1), 5-14.

Lara, M. A., Le, H. N., Letechipia, G., \& Hochhausen, L. (2009). Prenatal depression in Latinas in the US and Mexico. Maternal \& Child Health Journal, 13(4), 567-576.

Le, H. N., Perry, D. F., \& Stuart, E. A. (2011). Randomized controlled trial of a preventive intervention for perinatal depression in highrisk Latinas. Journal of Consulting and Clinical Psychology, 79(2), 135-141.

Levy, L. B., \& O'Hara, M. W. (2010). Psychotherapeutic interventions for depressed, low-income women: A review of the literature. Clinical Psychology Review, 30(8), 934-950.

Marcus, S., Lopez, J. F., McDonough, S., Mackenzie, M. J., Flynn, H., Neal, C. R., Jr., et al. (2011). Depressive symptoms during pregnancy: Impact on neuroendocrine and neonatal outcomes. Infant Behavior \& Development, 34(1), 26-34.

Martinez-Schallmoser, L., MacMullen, N. J., \& Telleen, S. (2005). Social support in Mexican American childbearing women. Journal of Obstetrics, Gynecology, and Neonatal Nursing, 34(6), 755-760.

Martinez-Schallmoser, L., Telleen, S., \& MacMullen, N. J. (2003). The effect of social support and acculturation on postpartum depression in Mexican American women. Journal of Transcultural Nursing, 14(4), 329-338.

McGarry, J., Kim, H., Sheng, X., Egger, M., \& Baksh, L. (2009). Postpartum depression and help-seeking behavior. Journal of Midwifery \& Women's Health, 54(1), 50-56.

Melville, J. L., Gavin, A., Guo, Y., Fan, M. Y., \& Katon, W. J. (2010). Depressive disorders during pregnancy: Prevalence and risk factors in a large urban sample. Obstetrics and Gynecology, 116(5), 1064-1070.

Miranda, J., Chung, J. Y., Green, B. L., Krupnick, J., Siddique, J., Revicki, D. A., et al. (2003). Treating depression in predominantly low-income young minority women: A randomized controlled trial. Journal of the American Medical Association, 290(1), 57-65.

Mora, P. A., Bennett, I. M., Elo, I. T., Mathew, L., Coyne, J. C., \& Culhane, J. F. (2009). Distinct trajectories of perinatal depressive symptomatology: Evidence from growth mixture modeling. American Journal of Epidemiology, 169(1), 24-32.

Muñoz, R. F., Le, H. N., Ippen, C. G., Diaz, M. A., Urizar, G. G., Soto, J., et al. (2007). Prevention of postpartum depression in low-income women: Development of the Mamas y Bebes/ mothers and babies course. Cognitive and Behavioral Practice, 14(1), 70-83.

O'Hara, M. W. (2009). Postpartum depression: What we know. Journal of Clinical Psychology, 65(12), 1258-1269. 
Orr, S. T., Blazer, D. G., James, S. A., \& Reiter, J. P. (2007). Depressive symptoms and indicators of maternal health status during pregnancy. Journal of Women's Health, 16(4), 535-542.

Pearson, R. M., Cooper, R. M., Penton-Voak, I. S., Lightman, S. L., \& Evans, J. (2010). Depressive symptoms in early pregnancy disrupt attentional processing of infant emotion. Psychological Medicine, 40(4), 621-631.

Perreira, K. M., Deeb-Sossa, N., Harris, K. M., \& Bollen, K. (2005). What are we measuring? An evaluation of the CES-D across race/ethnicity and immigrant generation. Social Forces, 83(4), $1567-1602$.

Perry, D. F., Ettinger, A. K., Mendelson, T., \& Le, H. N. (2011). Prenatal depression predicts postpartum maternal attachment in low-income Latina mothers with infants. Infant Behavior \& Development, 34(2), 339-350.

Radloff, L. S. (1977). The CES-D scale: A self-report depression scale for research in the general population. Applied Psychological Measurement, 1(3), 385-401.

Rich-Edwards, J. W., Kleinman, K., Abrams, A., Harlow, B. L., McLaughlin, T. J., Joffe, H., et al. (2006). Sociodemographic predictors of antenatal and postpartum depressive symptoms among women in a medical group practice. Journal of Epidemiology and Community Health, 60(3), 221-227.

Roberts, R. E., \& Sobhan, M. (1992). Symptoms of depression in adolescence: A comparison of Anglo, African, and Hispanic Americans. Journal of Youth and Adolescence, 21(6), 639-651.

Robertson, E., Grace, S., Wallington, T., \& Stewart, D. E. (2004). Antenatal risk factors for postpartum depression: A synthesis of recent literature. General Hospital Psychiatry, 26(4), 289-295.

Roman, L. A., Gardiner, J. C., Lindsay, J. K., Moore, J. S., Luo, Z. H., Baer, L. J., et al. (2009). Alleviating perinatal depressive symptoms and stress: A nurse-community health worker randomized trial. Archives of Women's Mental Health, 12(6), 379-391.

Sleath, B., West, S., Tudor, G., Perreira, K., King, V., \& Morrissey, J. (2005). Ethnicity and prenatal depression: Women's experiences and perspectives on communicating about their emotions and feelings during pregnancy. Patient Education and Counseling, 58(1), 35-40.

Spencer, M. S., Rosland, A. M., Kieffer, E. C., Sinco, B. R., Valerio, M. A., Palmisano, G., et al. (2011). Effectiveness of a community health worker intervention among African American and Latino adults with type 2 diabetes: A randomized controlled trial. American Journal of Public Health, 101(12), 2253-2260.

Spinelli, M. G., \& Endicott, J. (2003). Controlled clinical trial of interpersonal psychotherapy versus parenting education program for depressed pregnant women. American Journal of Psychiatry, $160(3), 555-562$.

Stacciarini, J. M. (2008). Focus groups: Examining a communitybased group intervention for depressed Puerto Rican women. Issues in Mental Health Nursing, 29(7), 679-700.

Substance Abuse and Mental Health Services Administration (SAMHSA). (2010, May/June). Overview of the affordable care act: What are the implications for behavioral health? SAMHSA News, $18,15$.

Surkan, P. J., Peterson, K. E., Hughes, M. D., \& Gottlieb, B. R. (2006). The role of social networks and support in postpartum women's depression: A multiethnic urban sample. Maternal and Child Health Journal, 10(4), 375-383.

Thornton, P. L., Kieffer, E. C., Salabarría-Peña, Y., Odoms-Young, A., Willis, S. K., Kim, H., et al. (2006). Weight, diet, and physical activity-related beliefs and practices among pregnant and postpartum Latino women: The role of social support. Maternal and Child Health Journal, 10(1), 95-104.

US Census Bureau. (2000). American FactFinder-Census 2000. Retrieved June 13, 2011, from http://factfinder.census.gov.

US Census Bureau. (2009). American FactFinder-American Community Survey 2005-2009. Retrieved June 13, 2011, from http://factfinder.census.gov.

Vega, W. A., Ang, A., Rodriguez, M. A., \& Finch, B. K. (2011). Neighborhood protective effects on depression in Latinos. American Journal of Community Psychology, 47(1-2), 114-126.

White, L. R., Kohout, F., Evans, D. A., Cornoni-Huntley, J., \& Ostfeld, A. M. (1986). Related health problems. In J. CornoniHuntley, D. B. Brock, A. M. Ostfeld, J. O. Taylor \& R. B. Wallace (Eds.), Established populations for epidemiological studies of the elderly: Resource data book (NIH Publication No. 86-2443) (pp. 129-165). Washington, DC: US Public Health Service.

Wojcicki, J. M., Holbrook, K., Lustig, R. H., Epel, E., Caughey, A. B., Muñoz, R. F., et al. (2011). Chronic maternal depression is associated with reduced weight gain in Latino infants from birth to 2 years of age. PLOS ONE, 6(2), e16737.

Yonkers, K. A., Ramin, S. M., Rush, A. J., Navarrete, C. A., Carmody, T., March, D., et al. (2001). Onset and persistence of postpartum depression in an inner-city maternal health clinic system. American Journal of Psychiatry, 158(11), 1856-1863.

Zayas, L. H., Jankowski, K. R. B., \& McKee, M. D. (2003). Prenatal and postpartum depression among low-income Dominican and Puerto Rican women. Hispanic Journal of Behavioral Sciences, 25(3), 370-385. 